\title{
Group Classification of a Generalized Lane-Emden System
}

\author{
Ben Muatjetjeja, ${ }^{1}$ Chaudry Masood Khalique, ${ }^{1}$ and Fazal Mahmood Mahomed ${ }^{2}$ \\ ${ }^{1}$ Department of Mathematical Sciences, International Institute for Symmetry Analysis and Mathematical Modelling, \\ North-West University, Mafikeng Campus, Private Bag X2046, Mmabatho 2735, South Africa \\ ${ }^{2}$ Centre for Differential Equations, Continuum Mechanics and Applications, School of Computational and Applied Mathematics, \\ University of the Witwatersrand, (Wits), Johannesburg 2050, South Africa
}

Correspondence should be addressed to Chaudry Masood Khalique; masood.khalique@nwu.ac.za

Received 11 September 2012; Accepted 25 November 2012

Academic Editor: Mehmet Pakdemirli

Copyright (C) 2013 Ben Muatjetjeja et al. This is an open access article distributed under the Creative Commons Attribution License, which permits unrestricted use, distribution, and reproduction in any medium, provided the original work is properly cited.

We perform the group classification of the generalized Lane-Emden system $x u^{\prime \prime}+n u^{\prime}+x H(v)=0, x v^{\prime \prime}+n v^{\prime}+x g(u)=0$, which occurs in many applications of physical phenomena such as pattern formation, population evolution, and chemical reactions. We obtain four cases depending on the values of $n$.

\section{Introduction}

The celebrated Lane-Emden equation

$$
\frac{d^{2} y}{d x^{2}}+\frac{n}{x} \frac{d y}{d x}+f(y)=0
$$

where $n$ is a real constant and $f(y)$ is a real-valued function of the variable $y$, has many applications in mathematical physics and astrophysics. Equation (1), for certain fixed values of $n$ and $f(y)$, models several phenomena such as the theory of stellar structure, the thermal behavior of a spherical cloud of gas, isothermal gaseous sphere, and the theory of thermionic currents [1-3]. Several methods for the solution and many applications of the Lane-Emden Equation (1) can be found in the literature. The interested reader is referred to [4] and the references therein. It is worth mentioning that Wong [5], in his review paper of 1975 , presented more than 140 references on this topic.

A natural extension of (1), called the generalized LaneEmden system [6], is given by

$$
\begin{aligned}
& \frac{d^{2} u}{d x^{2}}+\frac{n}{x} \frac{d u}{d x}+H(v)=0 \\
& \frac{d^{2} v}{d x^{2}}+\frac{n}{x} \frac{d v}{d x}+G(u)=0
\end{aligned}
$$

Such systems arise in the modeling of several physical phenomena, such as pattern formation, population evolution, chemical reactions, and so on [7], and in the past few years have attracted much attention. Various researchers have worked on existence and uniqueness results for the LaneEmden systems [8,9] and other related systems [10-12].

In [6] the authors studied Noether operators with respect to the standard Lagrangian of the generalized coupled LaneEmden system (2). They obtained seven cases out of which six cases resulted in Noether point symmetries. The first integrals corresponding to the Noether operators in each case were also constructed.

The objective of this paper is to perform the Lie group classification of the generalized Lane-Emden system (2). The paper is organized as follows. In Section 2, we calculate the equivalence transformations of the Lane-Emden system (2). We determine the principal Lie algebra and perform the group classification of system (2) in Section 3. Finally, concluding remarks are presented in Section 4.

\section{Equivalence Transformations}

An equivalence transformation (see, e.g., [13]) of the system (2) is an invertible transformation involving the variables $x$, $u$, and $v$ that map system (2) into itself, with possibly the form of the transformed functions being different from that 
of the original functions $H(v)$ and $G(u)$. We write system (2) as

$$
\begin{aligned}
& \frac{d^{2} u}{d x^{2}}+\frac{n}{x} \frac{d u}{d x}+H(v)=0, \\
& \frac{d^{2} v}{d x^{2}}+\frac{n}{x} \frac{d v}{d x}+G(u)=0, \\
& H_{x}=0, \quad H_{u}=0, \quad G_{x}=0, \quad G_{v}=0,
\end{aligned}
$$

where $u$ and $v$ are differential variables with independent variable $x$, and $H$ is a differential function of the independent variables $x$ and $v$, whereas $G$ is a differential function of the independent variables $x$ and $u$. We obtain the generators of the group of equivalence transformations as

$$
\begin{aligned}
Y= & \xi(x, u, v) \frac{\partial}{\partial x}+\eta^{1}(x, u, v) \frac{\partial}{\partial u} \\
& +\eta^{2}(x, u, v) \frac{\partial}{\partial v}+\mu^{1}(x, u, v, H, G) \frac{\partial}{\partial H} \\
& +\mu^{2}(x, u, v, H, G) \frac{\partial}{\partial G}
\end{aligned}
$$

We apply Lie's infinitesimal approach by using the prolongation of $Y$ to involve the derivatives in system (3) as, for example, in [14].

We summarize our results below.

Case $1(n \neq-1,1,3)$. In this case system (3) has the ninedimensional equivalence Lie algebra spanned by the equivalence generators

$$
\begin{aligned}
& X_{1}=x \frac{\partial}{\partial x}-2 H \frac{\partial}{\partial H}-2 G \frac{\partial}{\partial G}, \\
& X_{2}=u \frac{\partial}{\partial u}+H \frac{\partial}{\partial H}, \\
& X_{3}=v \frac{\partial}{\partial v}+G \frac{\partial}{\partial G}, \\
& X_{4}=\frac{\partial}{\partial u} \\
& X_{5}=\frac{\partial}{\partial v} \\
& X_{6}=x^{1-n} \frac{\partial}{\partial u} \\
& X_{7}=x^{1-n} \frac{\partial}{\partial v} \\
& X_{8}=x^{2} \frac{\partial}{\partial u}-2(1+n) \frac{\partial}{\partial H}, \\
& X_{9}=x^{2} \frac{\partial}{\partial v}-2(1+n) \frac{\partial}{\partial G}
\end{aligned}
$$

and hence the nine-parameter equivalence group is given by

$$
\begin{aligned}
& X_{1}: \bar{x}=e^{a_{1}} x, \quad \bar{u}=u, \quad \bar{v}=v, \\
& \bar{H}=e^{-2 a_{1}} H, \quad \bar{G}=e^{-2 a_{1}} G, \\
& X_{2}: \bar{x}=x, \quad \bar{u}=e^{a_{2}} u, \quad \bar{v}=v, \\
& \bar{H}=e^{a_{2}} H, \quad \bar{G}=G, \\
& X_{3}: \bar{x}=x, \quad \bar{u}=u, \quad \bar{v}=e^{a_{3}} v, \\
& \bar{H}=H, \quad \bar{G}=e^{a_{3}} G, \\
& X_{4}: \bar{x}=x, \quad \bar{u}=u+a_{4}, \\
& \bar{v}=v, \quad \bar{H}=H, \quad \bar{G}=G, \\
& X_{5}: \bar{x}=x, \quad \bar{u}=u, \quad \bar{v}=v+a_{5}, \\
& \bar{H}=H, \quad \bar{G}=G, \\
& X_{6}: \bar{x}=x, \quad \bar{u}=u+a_{6} x^{1-n}, \\
& \bar{v}=v, \quad \bar{H}=H, \quad \bar{G}=G, \\
& X_{7}: \bar{x}=x, \quad \bar{u}=u, \quad \bar{v}=v+a_{7} x^{1-n}, \\
& \bar{H}=H, \quad \bar{G}=G, \\
& X_{8}: \bar{x}=x, \quad \bar{u}=u+a_{8} x^{2}, \quad \bar{v}=v, \\
& \bar{H}=H-2(1+n) a_{8}, \quad \bar{G}=G, \\
& X_{9}: \bar{x}=x, \quad \bar{u}=u, \quad \bar{v}=v+a_{9} x^{2}, \\
& \bar{H}=H, \quad \bar{G}=G-2(1+n) a_{9} .
\end{aligned}
$$

Thus the composition of these transformations gives

$$
\begin{gathered}
\bar{x}=e^{a_{1}} x, \\
\bar{u}=e^{a_{2}}\left(u+a_{8} x^{2}+a_{6} x^{1-n}+a_{4}\right), \\
\bar{v}=e^{a_{3}}\left(v+a_{9} x^{2}+a_{7} x^{1-n}+a_{5}\right), \\
\bar{H}=e^{a_{2}-2 a_{1}}\left(H-2(1+n) a_{8}\right), \\
\bar{G}=e^{a_{3}-2 a_{1}}\left(G-2(1+n) a_{9}\right) .
\end{gathered}
$$

Case $2(n=-1)$. In this case system (3) has the ninedimensional equivalence Lie algebra spanned by the equivalence generators

$$
\begin{aligned}
& X_{1}=x \frac{\partial}{\partial x}-2 H \frac{\partial}{\partial H}-2 G \frac{\partial}{\partial G}, \\
& X_{2}=u \frac{\partial}{\partial u}+H \frac{\partial}{\partial H},
\end{aligned}
$$




$$
\begin{aligned}
& X_{3}=v \frac{\partial}{\partial v}+G \frac{\partial}{\partial G}, \\
& X_{4}=\frac{\partial}{\partial u}, \\
& X_{5}=\frac{\partial}{\partial v}, \\
& X_{6}=x^{2} \ln x \frac{\partial}{\partial u}-2 \frac{\partial}{\partial H}, \\
& X_{7}=x^{2} \ln x \frac{\partial}{\partial v}-2 \frac{\partial}{\partial G}, \\
& X_{8}=x^{2} \frac{\partial}{\partial u}, \\
& X_{9}=x^{2} \frac{\partial}{\partial v}
\end{aligned}
$$

and hence the nine-parameter equivalence group is given by

$$
\begin{aligned}
& X_{1}: \bar{x}=e^{a_{1}} x, \quad \bar{u}=u, \quad \bar{v}=v, \\
& \bar{H}=e^{-2 a_{1}} H, \quad \bar{G}=e^{-2 a_{1}} G, \\
& X_{2}: \bar{x}=x, \quad \bar{u}=e^{a_{2}} u, \quad \bar{v}=v, \\
& \bar{H}=e^{a_{2}} H, \quad \bar{G}=G, \\
& X_{3}: \bar{x}=x, \quad \bar{u}=u, \quad \bar{v}=e^{a_{3}} v, \\
& \bar{H}=H, \quad \bar{G}=e^{a_{3}} G, \\
& X_{4}: \bar{x}=x, \quad \bar{u}=u+a_{4}, \quad \bar{v}=v, \\
& \bar{H}=H, \quad \bar{G}=G, \quad \bar{v}, \quad \bar{v}=v+a_{5}, \\
& X_{5}: \bar{x}=x, \quad \bar{u}=u, \quad \bar{G}, \\
& \bar{H}=H, \quad \bar{G}=G, \quad \bar{u}=u+a_{6} x^{2} \ln x, \quad \bar{v}=v, \\
& X_{6}: \bar{x}=x, \quad \bar{u}, \quad \bar{v}=v+a_{7} x^{2} \ln x, \\
& \bar{H}=H-2 a_{6}, \bar{G}=G, \\
& X_{7}: \bar{x}=x, \quad \bar{u}=u, \quad \bar{G}=G-2 a_{7}, \\
& \bar{H}=H, \quad \bar{u}=v . \\
& X_{8}: \bar{x}=x, \quad \bar{u}=u+a_{8} x^{2}, \quad \bar{v}=v, \\
& \bar{H}=H, \quad \bar{G}=G, \\
& X_{9}: \bar{x}=x, \quad \bar{u}=u, \quad \bar{v}=v+a_{9} x^{2}, \\
& \bar{H}=H, \quad \bar{G}=G .
\end{aligned}
$$

Hence the composition of these transformations gives

$$
\begin{gathered}
\bar{x}=e^{a_{1}} x, \\
\bar{u}=e^{a_{2}}\left(u+a_{6} x^{2} \ln x+x^{2} a_{8}+a_{4}\right), \\
\bar{v}=e^{a_{3}}\left(v+a_{7} x^{2} \ln x+x^{2} a_{9}+a_{5}\right), \\
\bar{H}=e^{a_{2}-2 a_{1}}\left(H-2 a_{6}\right), \\
\bar{G}=e^{a_{3}-2 a_{1}}\left(G-2 a_{7}\right) .
\end{gathered}
$$

Case $3(n=1)$. In this case system (3) has the ninedimensional equivalence Lie algebra spanned by the equivalence generators

$$
\begin{aligned}
& X_{1}=x \frac{\partial}{\partial x}-2 H \frac{\partial}{\partial H}-2 G \frac{\partial}{\partial G}, \\
& X_{2}=u \frac{\partial}{\partial u}+H \frac{\partial}{\partial H}, \\
& X_{3}=v \frac{\partial}{\partial v}+G \frac{\partial}{\partial G}, \\
& X_{4}=\frac{\partial}{\partial u}, \\
& X_{5}=\frac{\partial}{\partial v}, \\
& X_{6}=\ln x \frac{\partial}{\partial u}, \\
& X_{7}=\ln x \frac{\partial}{\partial v}, \\
& X_{8}=x^{2} \frac{\partial}{\partial u}-4 \frac{\partial}{\partial H}, \\
& X_{9}=x^{2} \frac{\partial}{\partial v}-4 \frac{\partial}{\partial G}
\end{aligned}
$$

and hence the nine-parameter equivalence group is given by

$$
\begin{gathered}
X_{1}: \bar{x}=e^{a_{1}} x, \quad \bar{u}=u, \quad \bar{v}=v, \\
\bar{H}=e^{-2 a_{1}} H, \quad \bar{G}=e^{-2 a_{1}} G, \\
X_{2}: \bar{x}=x, \quad \bar{u}=e^{a_{2}} u, \quad \bar{v}=v, \\
\bar{H}=e^{a_{2}} H, \quad \bar{G}=G, \\
X_{3}: \bar{x}=x, \quad \bar{u}=u, \quad \bar{v}=e^{a_{3}} v, \\
\bar{H}=H, \quad \bar{G}=e^{a_{3}} G, \\
X_{4}: \bar{x}=x, \quad \bar{u}=u+a_{4}, \quad \bar{v}=v, \\
\bar{H}=H, \quad \bar{G}=G,
\end{gathered}
$$


TABLE 1 : Lie symmetries for $n \neq-1,1,3$, for various functions $H(v)$ and $G(u)$.

$H(v)$ arbitrary, $G(u)=c, c$ a constant

$X_{1}=\frac{\partial}{\partial u}, X_{2}=x^{(1-n)} \frac{\partial}{\partial u}, X_{3}=\left(c x^{2}+2 n v+2 v\right) \frac{\partial}{\partial u}$

$H(v)=d, d$ a constant, $G(u)$ arbitrary

$X_{1}=\frac{\partial}{\partial v}, X_{2}=x^{(1-n)} \frac{\partial}{\partial v}, X_{3}=\left(d x^{2}+2 n u+2 u\right) \frac{\partial}{\partial v}$

$H(v)=d, d$ a constant, $G(u)=\alpha+\beta u, \alpha$ and $\beta$ constants $(\beta \neq 0)$

$X_{1}=\frac{\partial}{\partial v}, X_{2}=x^{(1-n)} \frac{\partial}{\partial v}, X_{3}=\left(d x^{2}+2 n u+2 u\right) \frac{\partial}{\partial v}$,

$X_{4}=\beta x^{(3-n)} \frac{\partial}{\partial v}+(2 n-6) x^{(1-n)} \frac{\partial}{\partial u}$,

$X_{5}=(2 n+2) \frac{\partial}{\partial u}-\beta x^{2} \frac{\partial}{\partial v}$,

$X_{6}=\left(2 n^{2}+8 n+6\right) x \frac{\partial}{\partial x}-4\left(n^{2} u+3 d x^{2}+4 n u+3 u+n d x^{2}\right) \frac{\partial}{\partial u}+\left(\beta d x^{2}-2 \alpha n-6 \alpha\right) x^{2} \frac{\partial}{\partial v}$,

$X_{7}=\left(16 n x+4 n^{2} x+12 x\right) \frac{\partial}{\partial x}-(4 n+12) d x^{2} \frac{\partial}{\partial u}+\left(8 n^{2} v+32 n v+\beta d x^{4}+24 v\right) \frac{\partial}{\partial v}$

$H(v)=d, d$ a constant, $G(u)=\alpha+\beta u^{-p}, \alpha, p$, and $\beta$ constants $(\beta, p \neq 0)$

$X_{1}=\frac{\partial}{\partial v}, X_{2}=x^{(1-n)} \frac{\partial}{\partial v}$,

$X_{3}=(n x+x) \frac{\partial}{\partial x}+(2 n u+2 u) \frac{\partial}{\partial u}-\left(2 n p v-2 n v+\alpha p x^{2}+2 p v-2 v\right) \frac{\partial}{\partial v}$,

$X_{4}=\left(2 u+2 n u+d x^{2}\right) \frac{\partial}{\partial v}$

$H(v)=d, d$ a constant, $G(u)=\alpha+\beta e^{-k u}, \alpha, k$, and $\beta$ constants $(\beta, k \neq 0)$

$X_{1}=\frac{\partial}{\partial v}, X_{2}=x^{(1-n)} \frac{\partial}{\partial v}, X_{3}=\left(d x^{2}+2 n u+2 u\right) \frac{\partial}{\partial v}$,

$X_{4}=(2 n+2) \frac{\partial}{\partial u}-\left(\alpha k x^{2}+2 k n v+2 k v\right) \frac{\partial}{\partial v}$

$H(v)=d, d$ a constant, $G(u)=\alpha+\beta \ln u, \alpha$ and $\beta$ constants $(\beta \neq 0)$

$X_{1}=\frac{\partial}{\partial v}, X_{2}=x^{(1-n)} \frac{\partial}{\partial v}, X_{3}=\left(d x^{2}+2 n u+2 u\right) \frac{\partial}{\partial v}$,

$X_{4}=(x n+x) \frac{\partial}{\partial x}+(2 u+2 n u) \frac{\partial}{\partial u}+\left(2 n v-\beta x^{2}+2 v\right) \frac{\partial}{\partial v}$

$H(v)=a+b v, a$ and $b$ constants $(b \neq 0), G(u)=c, c$ constant

$X_{1}=\frac{\partial}{\partial u}, X_{2}=x^{(1-n)} \frac{\partial}{\partial u}, X_{3}=\left(c x^{2}+2 n v+2 v\right) \frac{\partial}{\partial u}$,

$X_{4}=b x^{(3-n)} \frac{\partial}{\partial u}+(2 n-6) x^{(1-n)} \frac{\partial}{\partial v}$,

$X_{5}=b x^{2} \frac{\partial}{\partial u}-(2 n+2) \frac{\partial}{\partial v}$,

$X_{6}=\left(b c x^{4}-32 n u-12 a x^{2}-24 u-8 n^{2} u-4 a n x^{2}\right) \frac{\partial}{\partial u}-\left(4 c n x^{2}+24 v+8 n^{2} v+32 n v+12 x^{2}\right) \frac{\partial}{\partial v}$,

$X_{7}=\left(16 n x+4 n^{2} x+12 x\right) \frac{\partial}{\partial x}+\left(8 n^{2} u+24 u+32 n u+b c x^{4}\right) \frac{\partial}{\partial u}-\left(12 c x^{2}+4 c n x^{2}\right) \frac{\partial}{\partial v}$

$H(v)=a+b v, a$ and $b$ constants $(b \neq 0), G(u)=\alpha+\beta u, \alpha$ and $\beta$ constants $(\beta \neq 0)$

$X_{1}=F(x) \frac{\partial}{\partial v}, X_{2}=W(x) \frac{\partial}{\partial u}, X_{3}=u \frac{\partial}{\partial u}+v \frac{\partial}{\partial v}$,

$X_{4}=b v \frac{\partial}{\partial u}+\beta u \frac{\partial}{\partial v}$

$H(v)=a+b v, a$ and $b$ constants $(b \neq 0), G(u)=\beta u^{-p}, \beta$ and $p$ constants $(\beta, p \neq 0)$

$X_{1}=(b x+b p x) \frac{\partial}{\partial x}+4 b u \frac{\partial}{\partial u}+(2 a+2 b v-2 a p-2 b p v) \frac{\partial}{\partial v}$

$H(v)=a+b v, a$ and $b$ constants $(b \neq 0), G(u)=\beta e^{-k u}, \beta$ and $k$ constants $(\beta, k \neq 0)$

$X_{1}=b k x \frac{\partial}{\partial x}+4 b \frac{\partial}{\partial u}-(2 a k+2 b k v) \frac{\partial}{\partial v}$ 
TABle 1: Continued.

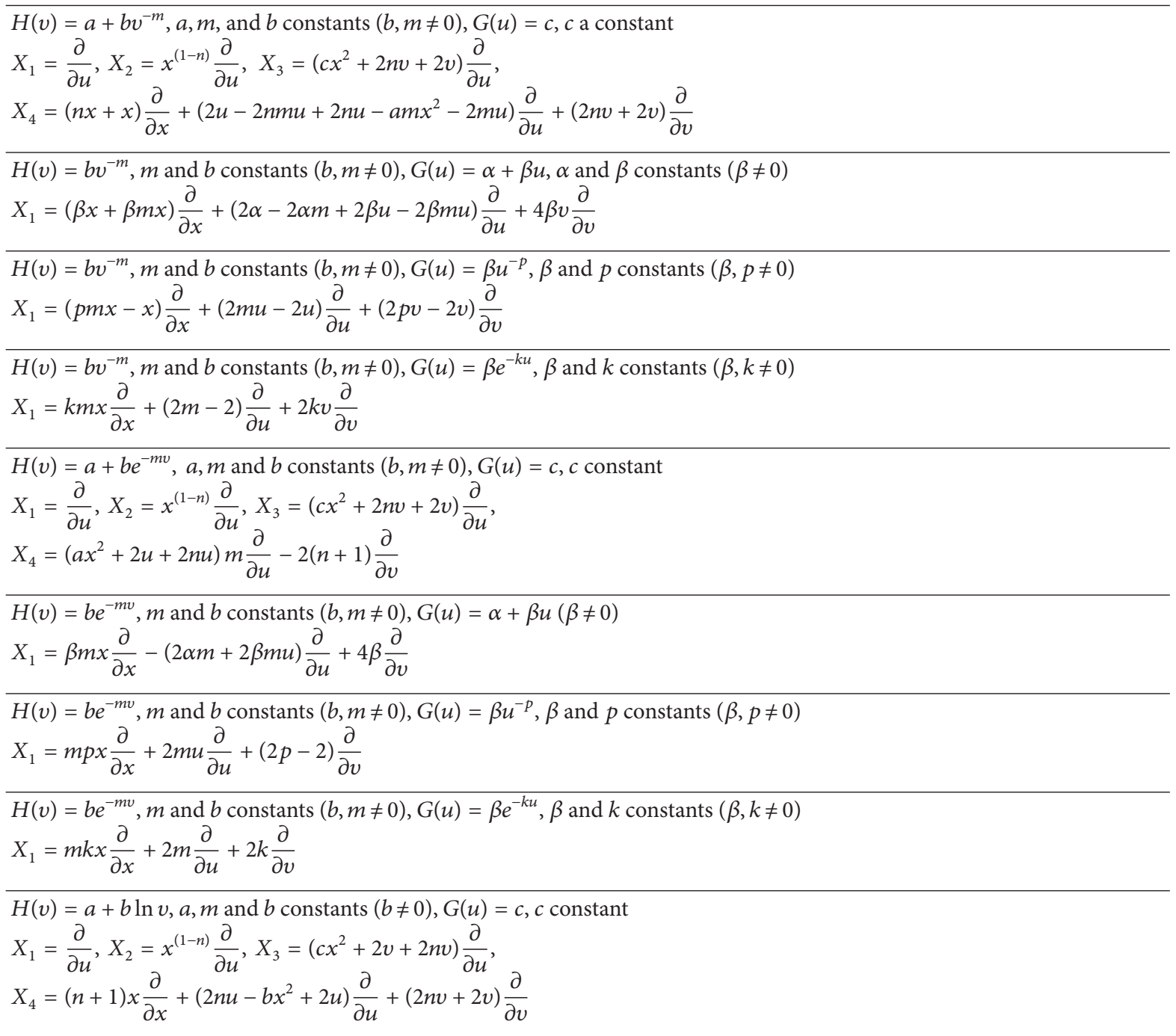

and so the composition of these transformations gives

$$
\begin{gathered}
\bar{x}=e^{a_{1}} x, \\
\bar{u}=e^{a_{2}}\left(u+a_{6} \ln x+a_{8} x^{2}+a_{4}\right), \\
\bar{v}=e^{a_{3}}\left(v+a_{7} \ln x+a_{9} x^{2}+a_{5}\right), \\
\bar{H}=e^{a_{2}-2 a_{1}}\left(H-4 a_{8}\right), \\
\bar{G}=e^{a_{3}-2 a_{1}}\left(G-4 a_{9}\right) .
\end{gathered}
$$

Case $4(n=3)$. In this case system (3) has the ten-dimensional equivalence Lie algebra spanned by the equivalence generators

$$
\begin{aligned}
& X_{1}=x \frac{\partial}{\partial x}-2 H \frac{\partial}{\partial H}-2 G \frac{\partial}{\partial G}, \\
& X_{2}=x^{-1} \frac{\partial}{\partial x}-2 x^{-2} u \frac{\partial}{\partial u}-2 x^{-2} v \frac{\partial}{\partial v},
\end{aligned}
$$


TABLE 2: Lie symmetries for $n=-1$, for various functions $H(v)$ and $G(u)$.

$H(v)$ arbitrary, $G(u)=c, c$ a constant

$X_{1}=\frac{\partial}{\partial u}, X_{2}=x^{2} \frac{\partial}{\partial u}, X_{3}=\left(c x^{2} \ln x+2 v\right) \frac{\partial}{\partial u}$

$H(v)=d, d$ a constant, $G(u)$ arbitrary

$X_{1}=\frac{\partial}{\partial v}, X_{2}=x^{2} \frac{\partial}{\partial v}, X_{3}=\left(d x^{2} \ln x+2 u\right) \frac{\partial}{\partial v}$

$H(v)=d, d$ a constant, $G(u)=\alpha+\beta u, \alpha$ and $\beta$ constants $(\beta \neq 0)$

$X_{1}=\frac{\partial}{\partial v}, X_{2}=x^{2} \frac{\partial}{\partial v}, X_{3}=\left(d x^{2} \ln x+2 u\right) \frac{\partial}{\partial v}, X_{4}=\beta x^{4} \frac{\partial}{\partial v}-8 x^{2} \frac{\partial}{\partial u}$,

$X_{5}=\beta x^{2} \ln x \frac{\partial}{\partial v}-2 \frac{\partial}{\partial u}$

$X_{6}=\left(4 d \beta x^{4} \ln x-32 \alpha x^{2} \ln x-3 \beta d x^{4}-64 v\right) \frac{\partial}{\partial v}-\left(32 x^{2} \ln x+64 u\right) \frac{\partial}{\partial u}$,

$X_{7}=16 x \frac{\partial}{\partial x}+\left(4 d \beta x^{4} \ln x-16 \alpha x^{2} \ln x-3 \beta d x^{4}\right) \frac{\partial}{\partial v}-\left(32 d x^{2} \ln x+32 u\right) \frac{\partial}{\partial u}$

$H(v)=d, d$ a constant, $G(u)=\alpha+\beta u^{-p}, \alpha, p$, and $\beta$ constants $(\beta, p \neq 0)$

$X_{1}=\frac{\partial}{\partial v}, X_{2}=x^{2} \frac{\partial}{\partial v}, X_{3}=\left(d x^{2} \ln x+2 u\right) \frac{\partial}{\partial v}$,

$X_{4}=x \frac{\partial}{\partial x}+2 u \frac{\partial}{\partial u}+\left(2 v-2 p v-\alpha p x^{2} \ln x\right) \frac{\partial}{\partial v}$

$H(v)=d, d$ a constant, $G(u)=\alpha+\beta e^{-k u}, \alpha, k$, and $\beta$ constants $(\beta, k \neq 0)$

$X_{1}=\frac{\partial}{\partial v}, X_{2}=x^{2} \frac{\partial}{\partial v}, X_{3}=\left(d x^{2} \ln x+2 u\right) \frac{\partial}{\partial v}$,

$X_{4}=2 \frac{\partial}{\partial u}-\left(\alpha k x^{2} \ln x+2 k v\right) \frac{\partial}{\partial v}$

$H(v)=d, d$ a constant, $G(u)=\alpha+\beta \ln u, \alpha$ and $\beta$ constants $(\beta \neq 0)$

$X_{1}=\frac{\partial}{\partial v}, X_{2}=x^{2} \frac{\partial}{\partial v}, X_{3}=\left(d x^{2} \ln x+2 u\right) \frac{\partial}{\partial v}$,

$X_{4}=x \frac{\partial}{\partial x}+2 u \frac{\partial}{\partial u}+\left(2 v-\beta x^{2} \ln x\right) \frac{\partial}{\partial v}$

$H(v)=a+b v, a$ and $b$ constants $(b \neq 0), G(u)=c, c$ constant

$X_{1}=\frac{\partial}{\partial u}, X_{2}=x^{2} \frac{\partial}{\partial u}, X_{3}=\left(c x^{2} \ln x+2 v\right) \frac{\partial}{\partial u}, X_{4}=c x^{2} \ln x \frac{\partial}{\partial u}-2 \frac{\partial}{\partial v}$,

$X_{5}=b x^{4} \ln x \frac{\partial}{\partial u}-8 x^{2} \frac{\partial}{\partial v}$,

$X_{6}=\left(4 b c x^{4} \ln x-32 a x^{2} \ln x-3 b c x^{4}-64 u\right) \frac{\partial}{\partial u}-\left(32 c x^{2} \ln x+64 v\right) \frac{\partial}{\partial v}$,

$X_{7}=16 x \frac{\partial}{\partial x}+\left(4 c d x^{4} \ln x-16 a x^{2} \ln x-3 b c x^{4}\right) \frac{\partial}{\partial u}-\left(32 c x^{2} \ln x+32 v\right) \frac{\partial}{\partial v}$

$H(v)=a+b v, a$ and $b$ constants $(b \neq 0), G(u)=\alpha+\beta u, \alpha$ and $\beta$ constants $(\beta \neq 0)$

$X_{1}=F(x) \frac{\partial}{\partial v}, X_{2}=W(x) \frac{\partial}{\partial u}, X_{3}=u \frac{\partial}{\partial u}+v \frac{\partial}{\partial v}$,

$X_{4}=b v \frac{\partial}{\partial u}+\beta u \frac{\partial}{\partial v}$

$H(v)=a+b v, a$ and $b$ constants $(b \neq 0), G(u)=\beta u^{-p}, \beta$ and $p$ constants $(\beta, p \neq 0)$

$X_{1}=(b x+b p x) \frac{\partial}{\partial x}+4 b u \frac{\partial}{\partial u}+(2 a+2 b v-2 a p-2 b p v) \frac{\partial}{\partial v}$

$H(v)=a+b v, a$ and $b$ constants $(b \neq 0), G(u)=\beta e^{-k u}, \beta$ and $k$ constants $(\beta, k \neq 0)$

$X_{1}=b k x \frac{\partial}{\partial x}+4 b \frac{\partial}{\partial u}-(2 a k+2 b k v) \frac{\partial}{\partial v}$

$H(v)=a+b v^{-m}, a, m$, and $b$ constants $(b, m \neq 0), G(u)=c, c$ a constant

$X_{1}=\frac{\partial}{\partial u}, X_{2}=x^{2} \frac{\partial}{\partial u}, X_{3}=\left(c x^{2} \ln x+2 v\right) \frac{\partial}{\partial u}$,

$X_{4}=x \frac{\partial}{\partial x}+\left(2 u-a m x^{2} \ln x-2 m u\right) \frac{\partial}{\partial u}+2 v \frac{\partial}{\partial v}$ 
TABLe 2: Continued.

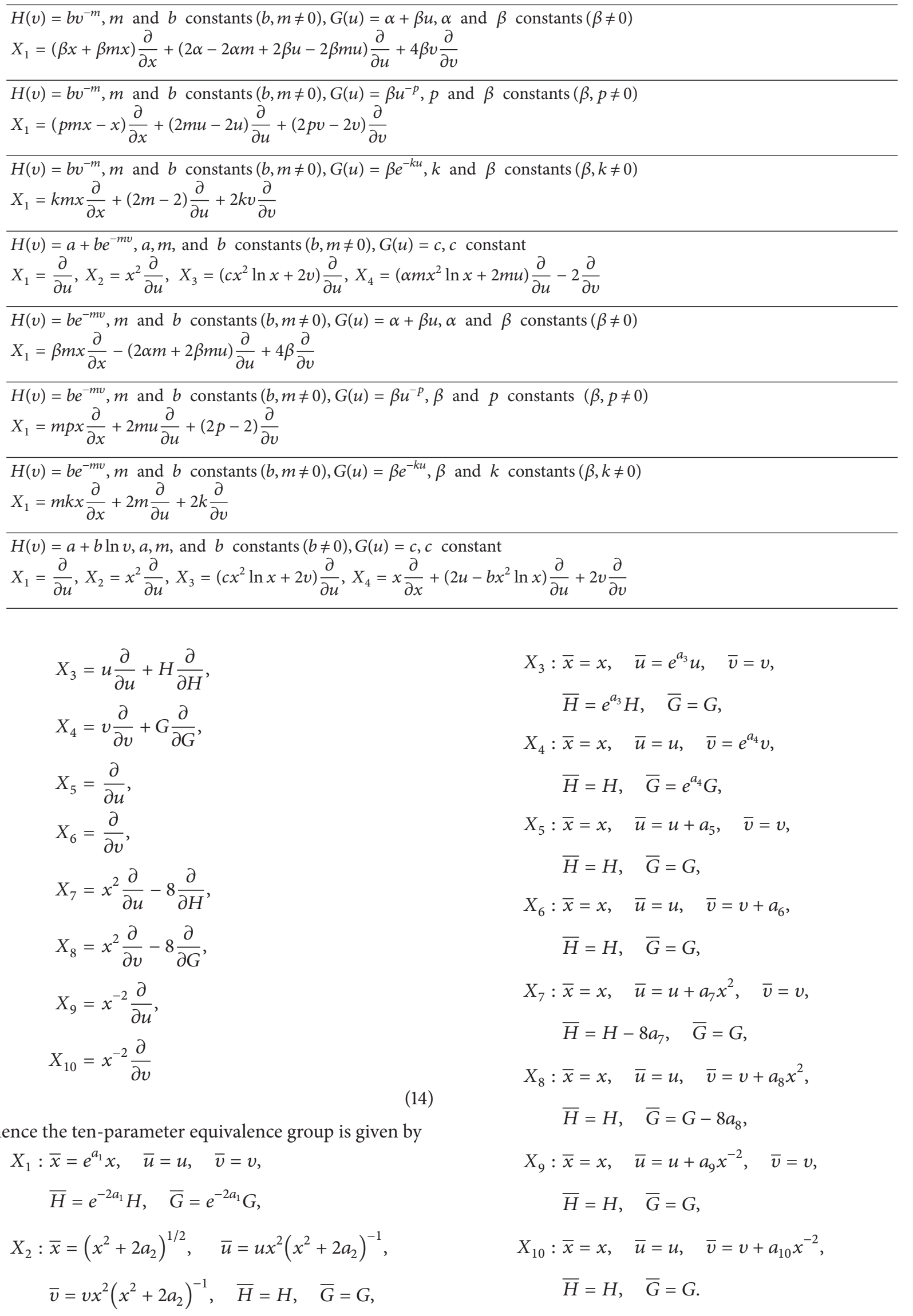


TABLE 3: Lie symmetries for $n=1$, for various functions $H(v)$ and $G(u)$.

$H(v)$ arbitrary, $G(u)=c, c$ a constant

$X_{1}=\frac{\partial}{\partial u}, X_{2}=\ln x \frac{\partial}{\partial u}, X_{3}=\left(c x^{2}+4 v\right) \frac{\partial}{\partial u}$

$H(v)=d, d$ a constant, $G(u)$ arbitrary

$X_{1}=\frac{\partial}{\partial v}, X_{2}=\ln x \frac{\partial}{\partial v}, X_{3}=\left(d x^{2} \ln x+4 u\right) \frac{\partial}{\partial v}$

$H(v)=d, d$ a constant, $G(u)=\alpha+\beta u, \alpha$ and $\beta$ constants $(\beta \neq 0)$

$X_{1}=\frac{\partial}{\partial v}, X_{2}=\ln x \frac{\partial}{\partial v}, X_{3}=\left(d x^{2} \ln x+4 u\right) \frac{\partial}{\partial v}, X_{4}=\beta x^{2} \frac{\partial}{\partial v}-4 \frac{\partial}{\partial u}$,

$X_{5}=\left(\beta x^{2} \ln x-\beta x^{2}\right) \frac{\partial}{\partial v}-4 \ln x \frac{\partial}{\partial u}$,

$X_{6}=\left(16 d x^{2}+64 u\right) \frac{\partial}{\partial u}+\left(16 \alpha x^{2}+64 v-\beta d x^{4}\right) \frac{\partial}{\partial v}$,

$X_{7}=16 x \frac{\partial}{\partial x}-\left(8 \alpha x^{2}-\beta d x^{4}\right) \frac{\partial}{\partial v}-\left(16 d x^{2}+32 u\right) \frac{\partial}{\partial u}$

$H(v)=d, d$ a constant, $G(u)=\alpha+\beta u^{-p}, \alpha, p$, and $\beta$ constants $(\beta, p \neq 0)$

$X_{1}=\frac{\partial}{\partial v}, X_{2}=\ln x \frac{\partial}{\partial v}, X_{3}=\left(d x^{2} \ln x+4 u\right) \frac{\partial}{\partial v}$,

$X_{4}=2 x \frac{\partial}{\partial x}+4 u \frac{\partial}{\partial u}-\left(4 v p+\alpha p x^{2}-4 v\right) \frac{\partial}{\partial v}$

$H(v)=d, d$ a constant, $G(u)=\alpha+\beta e^{-k u}, \alpha, k$, and $\beta$ constants $(\beta, k \neq 0)$

$X_{1}=\frac{\partial}{\partial v}, X_{2}=\ln x \frac{\partial}{\partial v}, X_{3}=\left(d x^{2}+4 u\right) \frac{\partial}{\partial v}$,

$X_{4}=4 \frac{\partial}{\partial u}-\left(\alpha k x^{2}+4 k v\right) \frac{\partial}{\partial v}$

$H(v)=d, d$ a constant, $G(u)=\alpha+\beta \ln u, \alpha$ and $\beta$ constants $(\beta \neq 0)$

$X_{1}=\frac{\partial}{\partial v}, X_{2}=\ln x \frac{\partial}{\partial v}, X_{3}=\left(d x^{2}+4 u\right) \frac{\partial}{\partial v}$,

$X_{4}=2 x \frac{\partial}{\partial x}+4 u \frac{\partial}{\partial u}+\left(4 v-\beta x^{2}\right) \frac{\partial}{\partial v}$

$H(v)=a+b v, a$ and $b$ constants $(b \neq 0), G(u)=c, c$ constant

$X_{1}=\frac{\partial}{\partial u}, X_{2}=\ln x \frac{\partial}{\partial u}, X_{3}=\left(c x^{2}+4 v\right) \frac{\partial}{\partial u}, X_{4}=4 \ln x \frac{\partial}{\partial v}-\left(b x^{2} \ln x-b x^{2}\right) \frac{\partial}{\partial u}$,

$X_{5}=b x^{2} \frac{\partial}{\partial u}-4 \frac{\partial}{\partial v}$,

$X_{6}=32 x \frac{\partial}{\partial x}-16 c x^{2} \frac{\partial}{\partial v}+\left(b c x^{4}+64 u\right) \frac{\partial}{\partial u}$,

$X_{7}=16 x \frac{\partial}{\partial x}-\left(8 a x^{2}-b c x^{4}\right) \frac{\partial}{\partial u}-\left(16 c x^{2}+32 v\right) \frac{\partial}{\partial v}$

$H(v)=a+b v, a$ and $b$ constants $(b \neq 0), G(u)=\alpha+\beta u, \alpha$ and $\beta$ constants $(\beta \neq 0)$

$X_{1}=F(x) \frac{\partial}{\partial v}, X_{2}=W(x) \frac{\partial}{\partial u}, X_{3}=u \frac{\partial}{\partial u}+v \frac{\partial}{\partial v}$,

$X_{4}=b v \frac{\partial}{\partial u}+\beta u \frac{\partial}{\partial v}$

$H(v)=a+b v, a$ and $b$ constants $(b \neq 0), G(u)=\beta u^{-p}, \beta$ and $p$ constants $(\beta, p \neq 0)$

$X_{1}=(b x+b p x) \frac{\partial}{\partial x}+4 b u \frac{\partial}{\partial u}+(2 a+2 b v-2 a p-2 b p v) \frac{\partial}{\partial v}$

$H(v)=a+b v, a$ and $b$ constants $(b \neq 0), G(u)=\beta e^{-k u}, \beta$ and $k$ constants $(\beta, k \neq 0)$

$X_{1}=b k x \frac{\partial}{\partial x}+4 b \frac{\partial}{\partial u}-(2 a k+2 b k v) \frac{\partial}{\partial v}$

$H(v)=a+b v^{-m}, a, m$, and $b$ constants $(b, m \neq 0), G(u)=c, c$ a constant

$X_{1}=\frac{\partial}{\partial u}, \quad X_{2}=\ln x \frac{\partial}{\partial u}, X_{3}=\left(c x^{2}+4 v\right) \frac{\partial}{\partial u}$,

$X_{4}=2 x \frac{\partial}{\partial x}+\left(4 u-a m x^{2}-4 m u\right) \frac{\partial}{\partial u}+4 v \frac{\partial}{\partial v}$ 
TABLE 3: Continued.

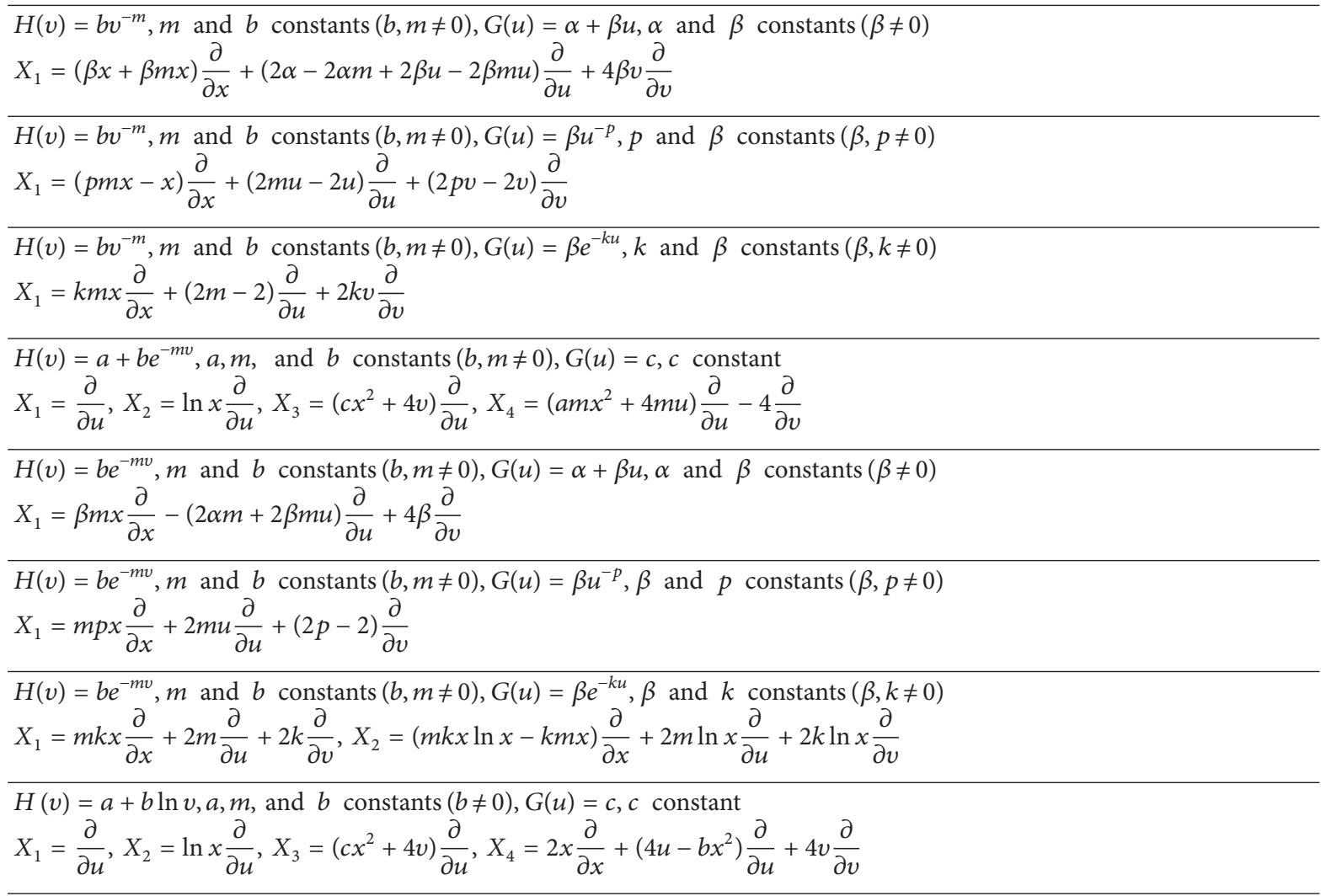

Therefore the composition of these transformations gives

$$
\begin{gathered}
\bar{x}=e^{a_{1}}\left(x^{2}+2 a_{2}\right)^{1 / 2}, \\
\bar{u}=e^{a_{3}}\left(x^{2}+2 a_{2}\right)^{-1}\left(x^{2} u+a_{9}+a_{7} x^{4}+a_{5} x^{2}\right), \\
\bar{v}=e^{a_{4}}\left(x^{2}+2 a_{2}\right)^{-1}\left(x^{2} v+a_{10}+a_{8} x^{4}+a_{6} x^{2}\right), \\
\bar{H}=e^{a_{3}-2 a_{1}}\left(H-8 a_{7}\right), \\
\bar{G}=e^{a_{4}-2 a_{1}}\left(G-8 a_{8}\right) .
\end{gathered}
$$

\section{Principal Lie Algebra and Lie Group Classification}

The generalized Lane-Emden system (2) admits a Lie point symmetry

$$
X=\xi(x, u, v) \frac{\partial}{\partial x}+\eta^{1}(x, u, v) \frac{\partial}{\partial u}+\eta^{2}(x, u, v) \frac{\partial}{\partial v}
$$

if

$$
\begin{aligned}
& \left.X^{[2]}\left(\frac{d^{2} u}{d x^{2}}+\frac{n}{x} \frac{d u}{d x}+H(v)\right)\right|_{(2)}=0, \\
& \left.X^{[2]}\left(\frac{d^{2} v}{d x^{2}}+\frac{n}{x} \frac{d v}{d x}+G(u)\right)\right|_{(2)}=0 .
\end{aligned}
$$

After some albeit tedious and lengthy calculations, the above determining equation gives

$$
\begin{gathered}
\xi=e(x), \\
\eta^{1}=k(x) u+l(x), \\
\eta^{2}=c(x) v+d(x), \\
-e^{\prime \prime}+\frac{n}{x} e^{\prime}-\frac{n}{x^{2}} e+2 k^{\prime}=0, \\
-e^{\prime \prime}+\frac{n}{x} e^{\prime}-\frac{n}{x^{2}} e+2 c^{\prime}=0, \\
(k u+l) G^{\prime}(u)+\left(2 e^{\prime}-c\right) G(u)+\frac{n}{x} d^{\prime}+d^{\prime \prime}=0, \\
(c v+d) H^{\prime}(v)+\left(2 e^{\prime}-k\right) H(v)+\frac{n}{x} l^{\prime}+l^{\prime \prime}=0 .
\end{gathered}
$$

Consequently, we conclude that the principal Lie algebra of (2) is trivial and the classifying relations are

$$
\begin{aligned}
& (\alpha u+\beta) G^{\prime}(u)+\gamma G(u)+\delta=0, \\
& (\theta v+\lambda) H^{\prime}(v)+\varphi H(v)+\omega=0,
\end{aligned}
$$

where $\alpha, \beta, \gamma, \delta, \theta, \lambda, \psi$, and $\omega$ are constants. 
TABLE 4: Lie symmetries for $n=3$, for various functions $H(v)$ and $G(u)$.

$H(v)$ arbitrary, $G(u)=c, c$ a constant

$X_{1}=\frac{\partial}{\partial u}, X_{2}=x^{-2} \frac{\partial}{\partial u}, X_{3}=\left(c x^{2}+8 v\right) \frac{\partial}{\partial u}$

$H(v)=d, d$ a constant, $G(u)$ arbitrary

$X_{1}=\frac{\partial}{\partial v}, X_{2}=x^{-2} \frac{\partial}{\partial v}, X_{3}=\left(d x^{2}+8 u\right) \frac{\partial}{\partial v}$

$H(v)=d, d$ a constant, $G(u)=\alpha+\beta u, \alpha$ and $\beta$ constants $(\beta \neq 0)$

$X_{1}=\frac{\partial}{\partial v}, X_{2}=x^{-2} \frac{\partial}{\partial v}, X_{3}=\left(d x^{2}+8 u\right) \frac{\partial}{\partial v}, X_{4}=\beta \ln x \frac{\partial}{\partial v}-2 x^{-2} \frac{\partial}{\partial u}$,

$X_{5}=\beta x^{2} \frac{\partial}{\partial v}-8 \frac{\partial}{\partial u}$,

$X_{6}=96 x \frac{\partial}{\partial x}-24 d x^{2} \frac{\partial}{\partial u}+\left(\beta d x^{4}+192 v\right) \frac{\partial}{\partial v}$,

$X_{7}=\left(192 u+24 d x^{4}\right) \frac{\partial}{\partial u}+\left(192 v-\beta d x^{2}+24 \alpha x^{2}\right) \frac{\partial}{\partial v}$

$H(v)=d, d$ a constant, $G(u)=\alpha+\beta u^{-p}, \alpha, p$, and $\beta$ constants $(\beta, p \neq 0)$

$X_{1}=\frac{\partial}{\partial v}, X_{2}=x^{-2} \frac{\partial}{\partial v}, X_{3}=\left(d x^{2}+8 u\right) \frac{\partial}{\partial v}$,

$X_{4}=4 x \frac{\partial}{\partial x}+8 u \frac{\partial}{\partial u}-\left(\alpha p x^{2}-8 v+8 p v\right) \frac{\partial}{\partial v}$

$H(v)=d, d$ a constant, $G(u)=\alpha+\beta e^{-k u}, \alpha, k$, and $\beta$ constants $(\beta, k \neq 0)$

$X_{1}=\frac{\partial}{\partial v}, X_{2}=x^{-2} \frac{\partial}{\partial v}, X_{3}=\left(d x^{2}+8 u\right) \frac{\partial}{\partial v}$,

$X_{4}=8 \frac{\partial}{\partial u}-\left(\alpha k x^{2}+8 k v\right) \frac{\partial}{\partial v}$

$H(v)=d, d$ a constant, $G(u)=\alpha+\beta \ln u, \alpha$ and $\beta$ constants $(\beta \neq 0)$

$X_{1}=\frac{\partial}{\partial v}, X_{2}=x^{-2} \frac{\partial}{\partial v}, X_{3}=\left(d x^{2}+8 u\right) \frac{\partial}{\partial v}$,

$X_{4}=4 x \frac{\partial}{\partial x}+8 u \frac{\partial}{\partial u}+\left(8 v-\beta x^{2}\right) \frac{\partial}{\partial v}$,

$X_{5}=x^{-1} \frac{\partial}{\partial x}-2 x^{-2} u \frac{\partial}{\partial u}-\left(2 x^{-2} v-\beta \ln x\right) \frac{\partial}{\partial v}$

$H(v)=a+b v, a$ and $b$ constants $(b \neq 0), G(u)=c, c$ constant

$X_{1}=\frac{\partial}{\partial u}, X_{2}=x^{-2} \frac{\partial}{\partial u}, X_{3}=\left(c x^{2}+8 v\right) \frac{\partial}{\partial u}, X_{4}=b \ln x \frac{\partial}{\partial u}-2 x^{-2} \frac{\partial}{\partial v}$,

$X_{5}=b x^{2} \frac{\partial}{\partial u}-8 \frac{\partial}{\partial v}$

$X_{6}=96 x \frac{\partial}{\partial x}+\left(b c x^{4}+192 u\right) \frac{\partial}{\partial u}-24 c x^{2} \frac{\partial}{\partial v}$,

$X_{7}=\left(24 a x^{2}+192 u-b c x^{4}\right) \frac{\partial}{\partial u}+\left(24 c x^{2}+192 v\right) \frac{\partial}{\partial v}$

$H(v)=a+b v, a$ and $b$ constants $(b \neq 0), G(u)=\alpha+\beta u, \alpha$ and $\beta$ constants $(\beta \neq 0)$

$X_{1}=F(x) \frac{\partial}{\partial v}, X_{2}=W(x) \frac{\partial}{\partial u}, X_{3}=u \frac{\partial}{\partial u}+v \frac{\partial}{\partial v}$,

$X_{4}=b v \frac{\partial}{\partial u}+\beta u \frac{\partial}{\partial v}$

$H(v)=a+b v, a$ and $b$ constants $(b \neq 0), G(u)=\beta u^{-p}, \beta$ and $p$ constants $(\beta, p \neq 0)$

$X_{1}=(b x+b p x) \frac{\partial}{\partial x}+4 b u \frac{\partial}{\partial u}+(2 a+2 b v-2 a p-2 b p v) \frac{\partial}{\partial v}$

$H(v)=a+b v, a$ and $b$ constants $(b \neq 0) G(u)=\beta e^{-k u}, \beta$ and $k$ constants $(\beta, k \neq 0)$

$X_{1}=b k x \frac{\partial}{\partial x}+4 b \frac{\partial}{\partial u}-(2 a k+2 b k v) \frac{\partial}{\partial v}$ 
TABle 4: Continued.

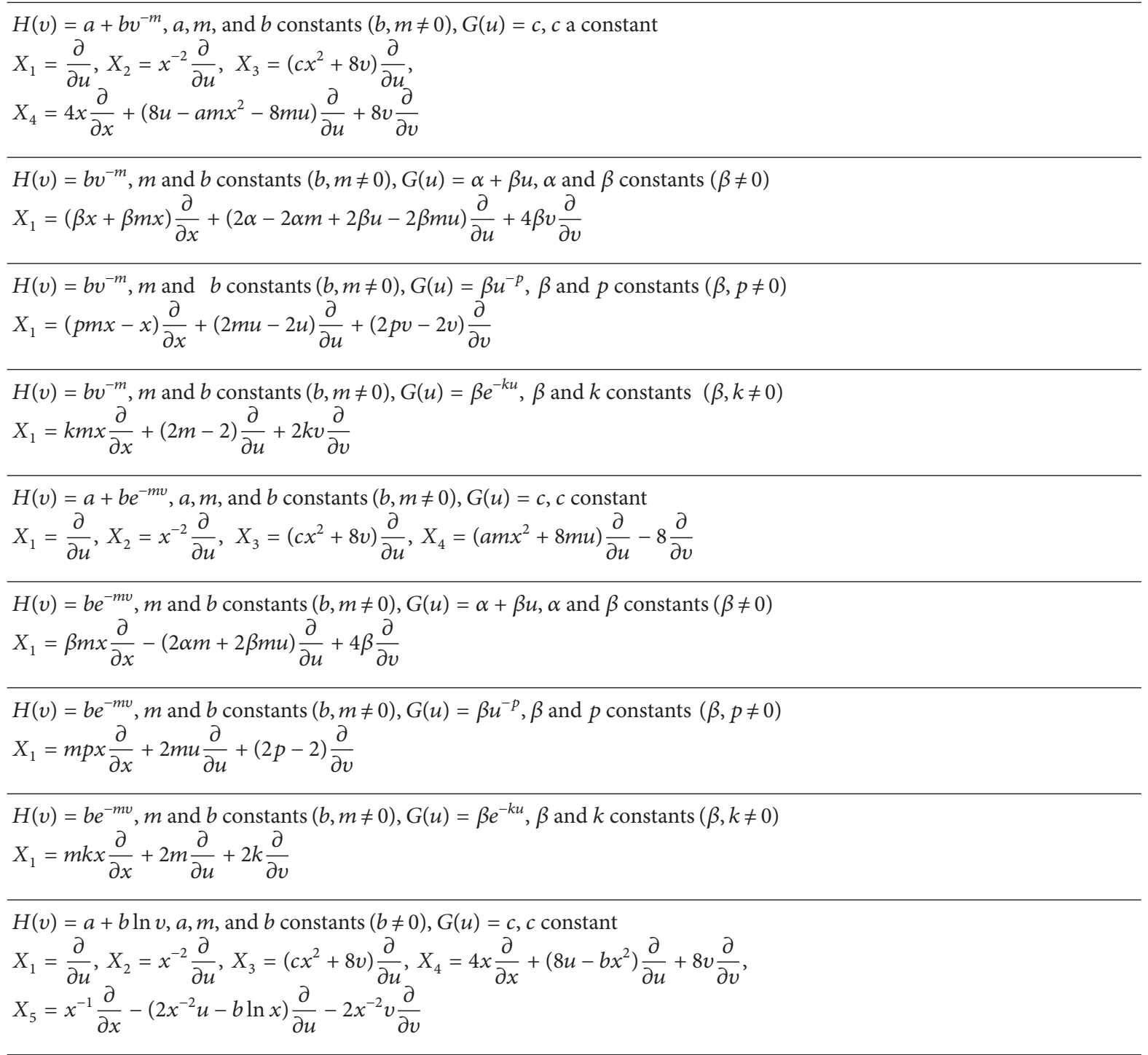

These classifying relations are invariant under the equivalence transformation (7) if

$$
\begin{gathered}
\bar{\alpha}=\alpha, \\
\bar{\beta}=\alpha\left(a_{8} x^{2}+a_{6} x^{1-n}+a_{4}\right)+\beta e^{-a_{2}}, \\
\bar{\gamma}=\gamma, \\
\bar{\delta}=\delta e^{2 a_{1}-a_{3}}-2 \gamma(1+n) a_{9}, \\
\bar{\theta}=\theta, \\
\bar{\lambda}=\theta\left(a_{9} x^{2}+a_{7} x^{1-n}+a_{5}\right)+\lambda e^{-a_{3}}, \\
\bar{\varphi}=\varphi \\
\bar{\omega}=\omega e^{2 a_{1}-a_{3}}-2 \varphi(1+n) a_{8} .
\end{gathered}
$$

The classifying relations (20) are also invariant under the equivalence transformation (10) if

$$
\begin{gathered}
\bar{\alpha}=\alpha, \\
\bar{\beta}=\alpha\left(a_{8} x^{2}+a_{6} x^{2} \ln x+a_{4}\right)+\beta e^{-a_{2}}, \\
\bar{\gamma}=\gamma, \\
\bar{\delta}=\delta e^{2 a_{1}-a_{3}}-2 \gamma a_{7}, \\
\bar{\theta}=\theta \\
\bar{\lambda}=\theta\left(a_{9} x^{2}+a_{7} x^{2} \ln x+a_{5}\right)+\lambda e^{-a_{3}}, \\
\bar{\varphi}=\varphi \\
\bar{\omega}=\omega e^{2 a_{1}-a_{2}}-2 \varphi a_{6} .
\end{gathered}
$$


It is also noted that the classifying relations (20) are invariant under the equivalence transformation (13) if

$$
\begin{gathered}
\bar{\alpha}=\alpha, \\
\bar{\beta}=\alpha\left(a_{6} \ln x+a_{8} x^{2}+a_{4}\right)+\beta e^{-a_{2}}, \\
\bar{\gamma}=\gamma, \\
\bar{\delta}=\delta e^{2 a_{1}-a_{3}}-4 \gamma a_{9}, \\
\bar{\theta}=\theta \\
\bar{\lambda}=\theta\left(a_{7} \ln x+a_{9} x^{2}+a_{5}\right)+\lambda e^{-a_{3}}, \\
\bar{\varphi}=\varphi \\
\bar{\omega}=\omega e^{2 a_{1}-a_{2}}-4 \varphi a_{8} .
\end{gathered}
$$

The classifying relations (20) are also invariant under the equivalence transformation (16) if

$$
\begin{gathered}
\bar{\alpha}=\alpha, \\
\bar{\beta}=\alpha\left(a_{9} x^{-2}+a_{7} x^{2}+a_{5}\right)+\beta\left(1+2 a_{2} x^{-2}\right) e^{-a_{3}}, \\
\bar{\gamma}=\gamma, \\
\bar{\delta}=\delta e^{2 a_{1}-a_{4}}-8 \gamma a_{8}, \\
\bar{\theta}=\theta, \\
\bar{\lambda}=\theta\left(a_{10} x^{-2}+a_{8} x^{2}+a_{6}\right)+\lambda\left(1+2 a_{2} x^{-2}\right) e^{-a_{4}}, \\
\bar{\varphi}=\varphi \\
\bar{\omega}=\omega e^{2 a_{1}-a_{3}}-8 \varphi a_{7} .
\end{gathered}
$$

The above relations are now used to find the nonequivalence forms of $H$ and $G$ and their corresponding Lie point symmetry. Several cases arise and are presented in Tables 1, 2, 3, and 4.

The Noether symmetries given in [6] from (25) to (44) always form a proper subalgebra of the Lie algebra that is obtained above. This can be seen from Tables 1, 2, 3, and 4 . However, in [6] the first integrals were also presented.

\section{Concluding Remarks}

We have studied a generalized coupled Lane-Emden system from the algebraic viewpoint. A complete group classification of the underlying system was performed. We showed that the generalized coupled Lane-Emden system admits a nine- or ten-dimensional equivalence Lie algebra. The principal Lie algebra, which was found to be trivial, had several possible extensions. We deduced the results for all possible cases of the values of $n$. There were in fact four cases that arose.

\section{Acknowledgment}

Two of the authors, B. Muatjetjeja and C. M. Khalique, would like to thank the Organizing Committee of "Symmetries,
Differential Equations, and Applications: Galois Bicentenary," (SDEA2012) Conference for their kind hospitality during the conference.

\section{References}

[1] S. Chandrasekhar, An Introduction to the Study of Stellar Structure, Dover, New York, NY, USA, 1957.

[2] H. T. Davis, Introduction to Nonlinear Differential and Integral Equations, Dover, New York, NY, USA, 1962.

[3] O. W. Richardson, The Emission of Electricity From Hot Bodies, Longmans, Green \& Co., London, UK, 2nd edition, 1921.

[4] B. Muatjetjeja and C. M. Khalique, "Exact solutions of the generalized Lane-Emden equations of the first and second kind," Pramana-Journal of Physics, vol. 77, no. 3, pp. 545-554, 2011.

[5] J. S. W. Wong, "On the generalized Emden-Fowler equation," SIAM Review, vol. 17, pp. 339-360, 1975.

[6] B. Muatjetjeja and C. M. Khalique, "Lagrangian approach to a generalized coupled Lane-Emden system: symmetries and first integrals," Communications in Nonlinear Science and Numerical Simulation, vol. 15, no. 5, pp. 1166-1171, 2010.

[7] H. Zou, "A priori estimates for a semilinear elliptic system without variational structure and their applications," Mathematische Annalen, vol. 323, no. 4, pp. 713-735, 2002.

[8] J. Serrin and H. Zou, "Non-existence of positive solutions of Lane-Emden systems," Differential and Integral Equations, vol. 9, no. 4, pp. 635-653, 1996.

[9] J. Serrin and H. Zou, "Existence of positive solutions of the Lane-Emden system," Atti del Seminario Matematico e Fisico dell'Università di Modena, vol. 46, supplement, pp. 369-380, 1998.

[10] Y.-W. Qi, “The existence of ground states to a weakly coupled elliptic system," Nonlinear Analysis: Theory, Methods \& Applications, vol. 48, no. 6, pp. 905-925, 2002.

[11] R. Dalmasso, "Existence and uniqueness of solutions for a semilinear elliptic system," International Journal of Mathematics and Mathematical Sciences, no. 10, pp. 1507-1523, 2005.

[12] Q. Dai and C. C. Tisdell, "Nondegeneracy of positive solutions to homogeneous second-order differential systems and its applications," Acta Mathematica Scientia. Series B, vol. 29, no. 2, pp. 435-446, 2009.

[13] N. H. Ibragimov, "Small effects in physics hinted by the Lie group philosophy: are they observable? I. From Galilean principle to heat diffusion," Lie Groups and Their Applications, vol. 1, no. 1, pp. 113-123, 1994.

[14] L. V. Ovsiannikov, Group Analysis of Differential Equations, Academic Press, New York, NY, USA, 1982. 


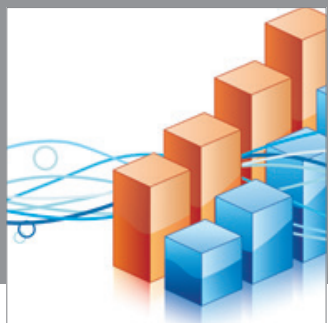

Advances in

Operations Research

mansans

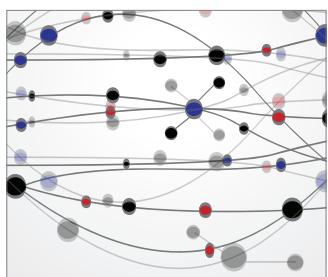

The Scientific World Journal
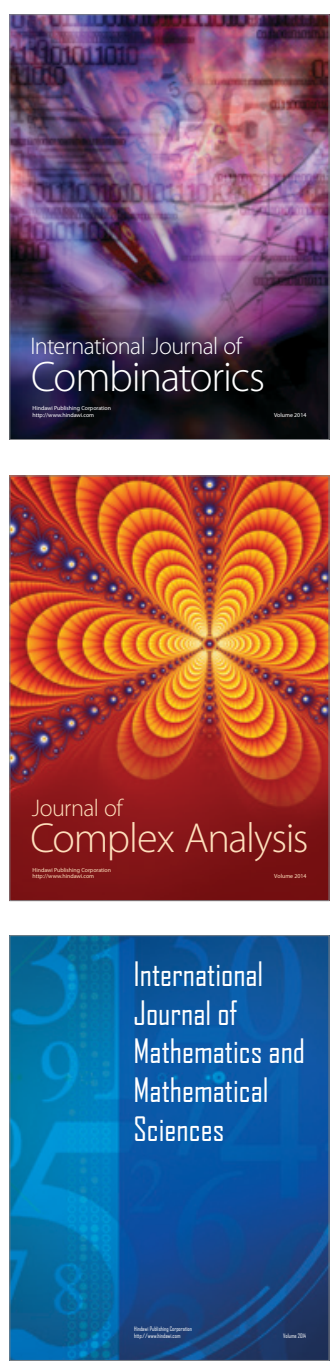
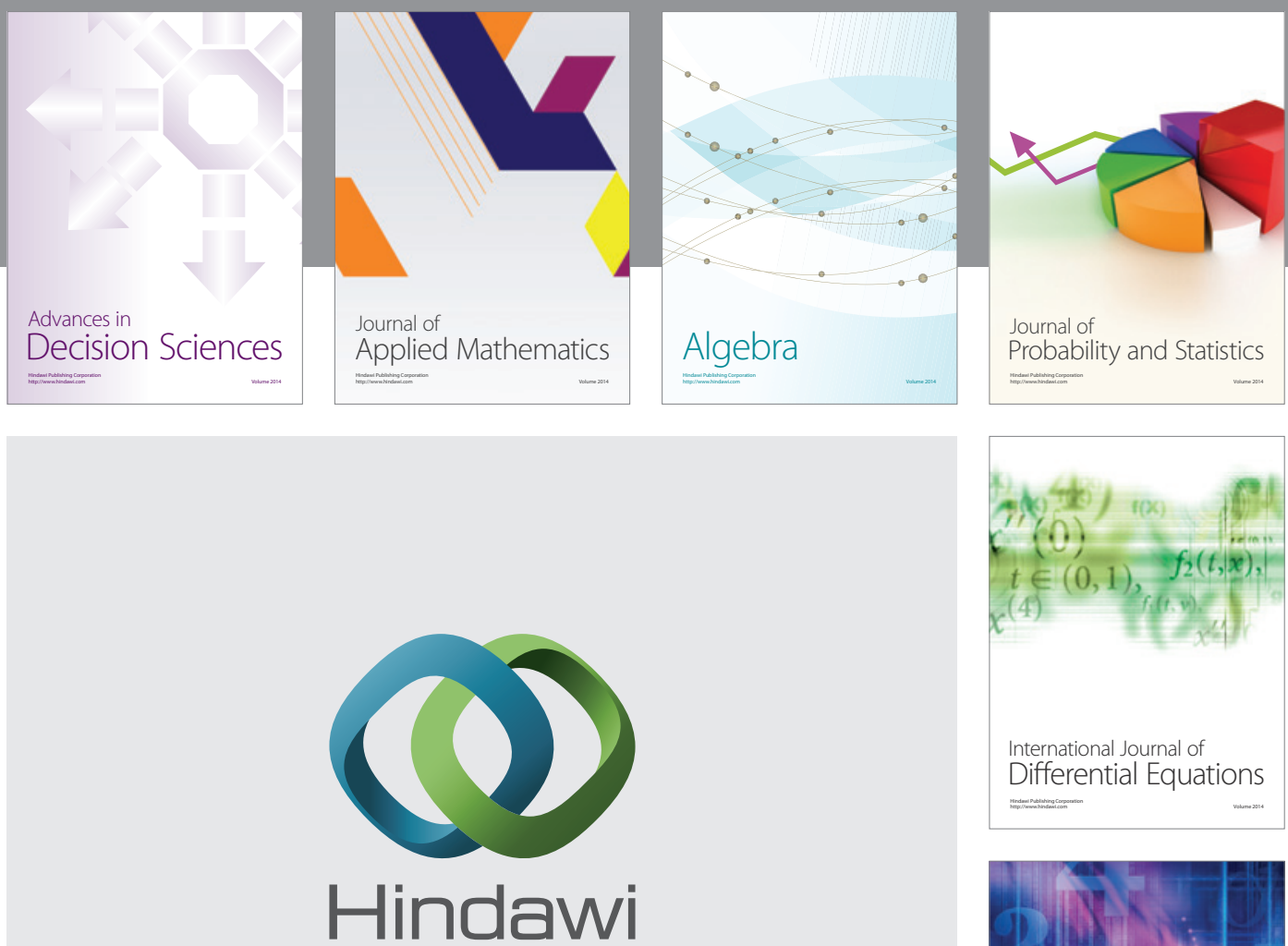

Submit your manuscripts at http://www.hindawi.com
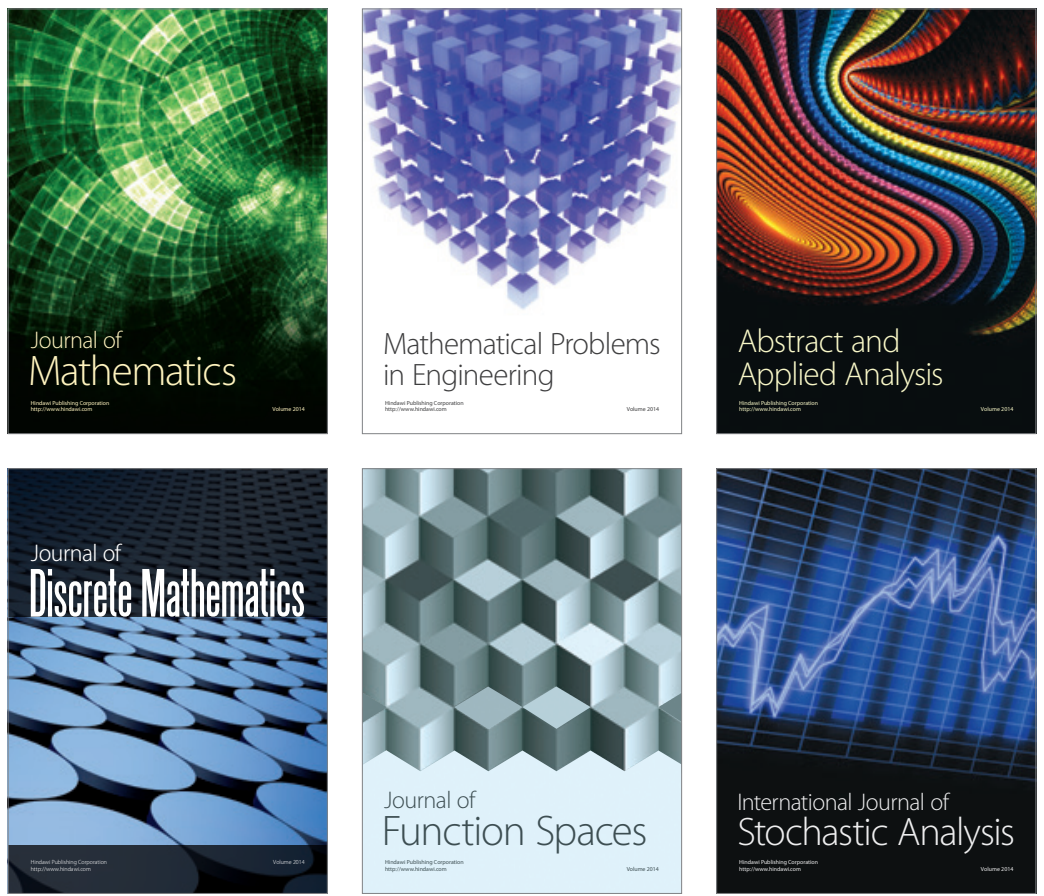

Journal of

Function Spaces

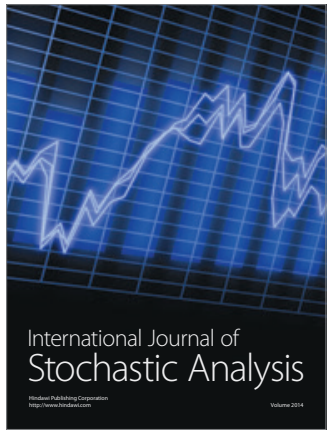

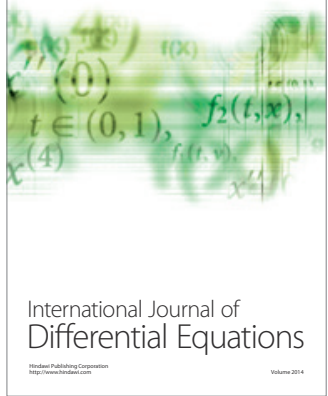
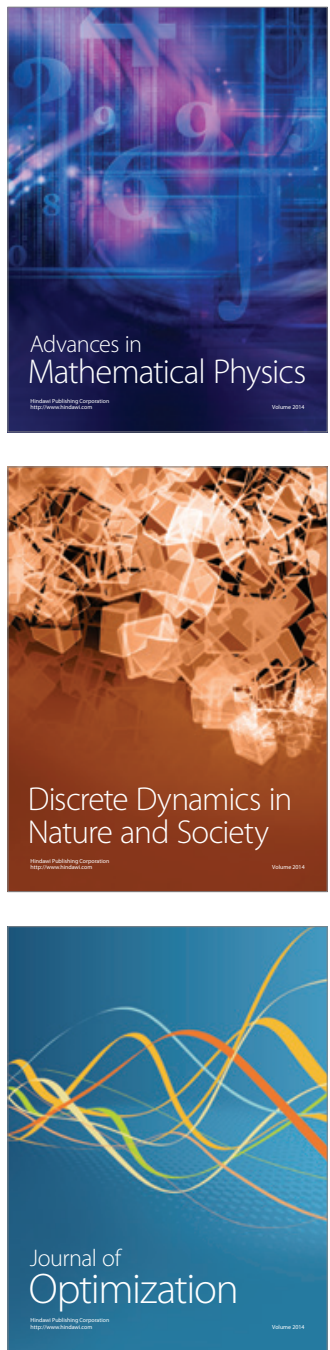